Article

\title{
Body Area Networks in Healthcare: A Brief State of the Art
}

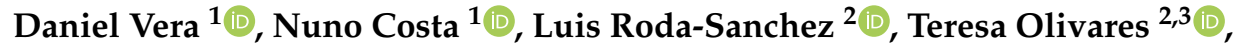 \\ Antonio Fernández-Caballero ${ }^{2,3}$ (iD) and Antonio Pereira $1, *$ (D) \\ 1 Polytechnic Institute of Leiria, Computer Science and Communications Research Centre, Campus 2, \\ Morro do Lena-Alto do Vieiro, Apartado 4163, 2411-901 Leiria, Portugal \\ 2 Instituto de Investigación en Informática,Universidad de Castilla-La Mancha, 02071 Albacete, Spain \\ 3 Departamento de Sistemas Informáticos, Universidad de Castilla-La Mancha, 02071 Albacete, Spain \\ * Correspondence: apereira@ipleiria.pt; Tel.: +351-244-820-300
}

Received: 24 May 2019; Accepted: 6 August 2019; Published: 8 August 2019

\begin{abstract}
A body area network (BAN) comprises a set of devices that sense their surroundings, activate and communicate with each other when an event is detected in its environment. Although BAN technology was developed more than 20 years ago, in recent years, its popularity has greatly increased. The reason is the availability of smaller and more powerful devices, more efficient communication protocols and improved duration of portable batteries. BANs are applied in many fields, healthcare being one of the most important through gathering information about patients and their surroundings. A continuous stream of information may help physicians with making well-informed decisions about a patient's treatment. Based on recent literature, the authors review BAN architectures, network topologies, energy sources, sensor types, applications, as well as their main challenges. In addition, the paper focuses on the principal requirements of safety, security, and sustainability. In addition, future research and improvements are discussed.
\end{abstract}

Keywords: body area networks; state of the art; healthcare; IEEE 802.15.6

\section{Introduction}

Improvements in several aspects related to quality of life like public access to physicians, medication, good nutrition and safety have produced an increment in the longevity of the population around the world. According to the United Nations, the population aged 60 years and over is estimated at 962 million in 2017 and the growth rate is about 3 percent per year [1]. The population aged 60 and over is expected to be around 2.1 billion in year 2050 and 3.1 billion in 2100 . Notice that Japanese aging adults have reached $27 \%$ of the population in 2017 . Unfortunately, such an increase supposes various inconveniences. For instance, this also means that the percentage of the working population is decreasing each year. For this reason, many governments are experiencing monetary difficulties to cover social security and healthcare costs.

In addition, people suffering from chronic diseases is increasing worldwide [2]. In year 2001, 46\% of reported deaths in the world were caused by chronic diseases. The World Health Organization predicts that deaths caused by chronic diseases will increase to $57 \%$ by year 2020 . Diabetes, obesity and cardiovascular diseases, especially in the aging population, cause almost half of the deaths produced by chronic diseases. These diseases affect a great amount of the population, even manifesting earlier in life. Therefore, early detection is imperative to fight against these diseases. Precisely, this can be achieved using noninvasive devices to monitor the health status through detecting and preventing chronic diseases based on a body area network (BAN). 
A BAN is formed by tiny devices which collect and transmit several kinds of information from multiple sources. BANs are applied in many fields, although some of the most significant application areas are healthcare and medicine. For this reason, BAN applications are usually divided into medical and non-medical applications [3]. In the first case, BANs are used to monitor patients suffering from chronic illnesses such as cardiovascular diseases, asthma or diabetes, among others. In this group of applications, BANs monitor the patients' vital signs permanently and, when a mishap occurs, it alerts the emergency services. The information is available at any time of the day. This helps the medical doctors to provide an accurate diagnosis and treatment. BANs can also help in cases of body rehabilitation and disability support [4].

Not surprisingly, there are many high quality surveys related to BANs that have been published in the last years. In 2011, two early surveys [5,6] focused on BAN/WBAN (wireless BAN) communication types, sensor devices, physical and data link layers, quality of service and security. Afterwards, a new survey [7] showed interest in intrabody communication (IBC) as alternative wireless communication technology, demonstrating mathematical models, IBC transceiver designs and core fundamentals. That same year, the IEEE 802.15.6 standard was used to review on-body, in-body and off-body propagation models in WBANs [8]. Next, an overview of the main applications, technologies, standards, issues and the future of WBANs was reported [9]. The authors also included some real cases and experimental studies.

Furthermore, during the last three years, surveys of specific issues within BANs have also been delivered. For instance, security is a fundamental aspect in any network where devices are susceptible to be attacked on sensitive information. This is the reason why many researchers orient their work to the development of techniques to avoid attacks in BANs. An overview of security in BANs, where the authors focused on key agreement schemes and methods used to evaluate security and performance have been introduced [10]. Similarly, there is a review on reliability and fault tolerance in WBAN solutions [11]. There is also a very recent paper that reviews the state-of-the-art of network architecture topologies and applications in Internet of Things (IoT)-based healthcare solutions [12]. Moreover, the authors analyze security, resource management, quality of service (QoS), real-time monitoring and other problematic factors in WBAN-based healthcare systems. Another paper surveys provides a taxonomy on QoS-based routing in WBANs [13]. Very recently, researchers conducted a review of WBAN applications and network architecture, routing protocols and data analysis techniques and how these aspects influence ubiquitous health monitoring [14].

In our humble opinion, this paper fills a current gap in BAN reviews. The focus of this paper is to offer a comprehensive brief state of the art to the overall readership interested in healthcare applications based on BANs. The remainder of the paper is organized as follows. Section 2 provides a short tutorial of BAN technology showing general features and requirements of these networks. Afterwards, the explanation is decomposed in Section 3, where physical layer aspects are treated, Section 4, in which data link requirements are afforded, possible network layer configurations and middleware layer issues in Section 5. Finally, in Section 6, important cross-layer properties are detailed. These sections take into consideration different approaches according to IEEE 802.15.16 standard, safety issues, privacy and security, and sustainability. Moreover, included in the physical layer, a variety of wearable sensors (mechanical, chemical, electrical and optical) are explored, explaining their pros and cons, and including a compilation of their utility in BAN healthcare applications. Finally, Section 7 shows current challenges in this field, and Section 8 presents the conclusions of this investigation as well as some future trends.

\section{Body Area Networks: Main Features and Requirements}

A BAN is an evolution of a wireless sensor network (WSN). WSNs were first used for personal devices like cellphones, laptops, and cameras. These devices form a personal area network (PAN). The same concept is applied to a BAN. A BAN is a network of nodes with sensors and actuators which communicate with each other. 
In addition to addressing aspects related to the lower layers of the OSI model, the design of BAN solutions should consider many general requirements such as safety, privacy and security and sustainability to ensure proper operation. However, in order to provide the best user experience, there are other aspects, e.g., quality of service and usability, which should be considered as well. A brief description of these issues is [15]:

- Safety: These types of networks, which are in direct contact with the body, must be designed to ensure they are safe for the end user. Hence, this is a critical point in the development of BAN devices, especially when medical applications are being developed, as parameters are measured in sensitive parts.

- Privacy and security: BAN applications collect a great amount of sensitive information that must only be accessible to authorized people. Unauthorized use of private information may lead to abuse and discrimination. BAN solutions that have access or generate sensitive information need to implement authentication and encryption. However, security policies should not overhead the energy consumption of the BAN nodes.

- Sustainability: Finding a balance between energy consumption and energy source is mandatory in BAN products. Energy supply must be available when required to achieve a good performance of the BAN solution. Energy-supply models are divided into two groups: (1) the node is supplied directly by the harvesting energy source (SSCS), and (2) the harvesting source saves energy into a battery and the battery supplies energy to the node (SBCS). SSCS involves connecting the energy source (solar panel, wind turbine, etc.) to the computing unit without any energy storage in between. This configuration must be calibrated to match the power supply and the demand in order to be sustainable. The SBCS uses a battery to store energy for later use, which is useful in situations where the power supply is not available at any time, like solar power in the night. In SBCS, the energy generated must be in balance with the energy that the battery can store to reduce waste. Testing the energy consumption of nodes helps to choose the correct energy source needed for the deployment.

- Quality of service: Each type of BAN application has a different definition of quality of service (QoS) depending on the services offered. Some elements to measure QoS are latency, jitters, and bandwidth of the network, stats of data transmission, errors and useful information sent in a period of time. In any case, QoS includes adaptability, timeliness, reliability, robustness and credibility for most applications. QoS can be separated into two cases, namely application and network [16]. The application perspective refers to the quality of the user interface (UI). The UI must be understandable and meet all the user's requirements. The network case measures the quality of the application's information provider (latency, reliability, error management, etc.).

- Usability: This is another requirement that has to be considered when developing BAN applications. Usability refers to how easily a person without information and communication technology (ICT) knowledge sets up the solution.

\section{Physical Layer Requirements}

\subsection{Properties According to IEEE 802.15.16 Standard}

Standards are developed so that industries and companies generate customer trust and enhance safety in their products. Standards help in the production of items which interact with each other regardless of their manufacturers. Standards define rules, protocols and other important parameters to ensure that products are in compliance with certain technical specifications [17]. The IEEE TG6 published the 802.15.6 standard on February 2012. This standard provides guidelines for BANs. IEEE 802.15.6 defines the physical (PHY) and data link layers. The purpose of 802.15 .6 is to provide a standard for a short range, highly reliable and low-power wireless communication close to or inside the human body. 
The standard IEEE 802.15.6 defines three PHY layers. These layers are narrow band PHY (NB-PHY), ultra-wide band PHY (UWB-PHY) and human body communication (HBC-PHY) [9].

NB-PHY was designed for wireless medical applications, divided into two groups: wearable and implantable. In the wearable group, the cables in a medical solution are replaced with highly reliable wireless communication methods, and one or both ends of the links are on the human body. The implantable group has a frequency band but not a standard yet. In order to support transmission and reception, a device must use one of the following bands: (a) for implantable devices, 402-405 MHz, and (b) for wearable devices, $420-450 \mathrm{MHz}, 863-870 \mathrm{MHz}, 902-928 \mathrm{MHz}, 950-958 \mathrm{MHz}$, 2360-2400 MHz, 2400-2483.5 MHz.

The ultra-wide band (UWB) layer offers high quality, low complexity, and ultra-low power operations. This layer is divided into two technologies: impulse radio (IR-UWB) and wide-band frequency modulation (FM-UWB). The band in this layer is classified into two groups, low (3.25-4.75 GHz) and high (6.6-10.25 GHz), each group being divided into $500 \mathrm{MHz}$ bandwidths. UWB-PHY applications have two operation modes. There is a default mode where IR-UWB is mandatory, while FM-UWB is optional. The default mode can be used in medical and non-medical applications. The second is a high quality of service mode, which is used in high priority medical applications. Therefore, FM-UWB is required to achieve a high quality of service.

Lastly, HBC-PHY uses the human body for exchanging information. It uses an electrode for transmitting or receiving an electrical signal from body to device. The operation band of this layer is $21 \mathrm{MHz}$ with a bandwidth of $5.25 \mathrm{MHz}$.

\subsection{Safety Issues in PHY Layer}

BAN safety is an important subject, especially in medical applications. The ISO 60601 standard [18] for medical devices defines what hazards a patient might experience when using these kinds of devices. The standard presents a list of aspects that could harm a patient: radiation, heat dissipation, biocompatibility, software errors, mechanical problems, and electric shocks. The principal objective is not harming the patient. Patient safety in medical BAN applications is a crucial point. Therefore, it has to be analyzed in the same way as the technical requirements. There are three perspectives to address when developing BAN systems:

- All possible critical events that may occur must be handled with a methodological approach. If a characteristic of the solution can cause damage to the user, it has to be tested first without living test subjects like human skin alternatives and always monitor hazardous radiations. A correct mitigation of possible hazardous situations saves patients' lives.

- BAN solutions use networking to communicate all data collected from the peripheral nodes with the purpose of achieving the solution goal. In medical applications, a networking failure can produce a fatal error. The developers must analyze the impact of delays and package loss in critical operations. Through the results of the analysis, the developers must improve the design of the solution mitigating the possible problems in critical operations.

- Software is the core of medical applications. Any failure in software is going to cause a malfunction that can harm the patient. The software has to be reviewed eliminating coding errors and testing all possible events that it must confront. Given the continuous interaction, it is important for the software to offer a way to receive feedback from the human body in order to prevent harm to the patient.

There are several options to ensure safety in all cases discussed before. For the purpose of assuring that BAN devices are safe to patients, experiments testing the effects of devices on human physiology are performed. These experiments are designed to mitigate the possible side effects to the subjects. Hardware models are also designed to emulate a part of the body in the case of medical applications. It is possible to test the device software and the control system through these models. Researchers must ensure that the software on BAN devices is working correctly by designing a large set of test cases. 
These tests cover a wide variety of plausible scenarios that the devices might encounter. The use of automaton devices helps to analyze the behavior of the BAN applications to provide safety guarantees.

\section{Model-Based Engineering}

Model-based engineering (MBE) is used to evaluate the safety in BAN systems. A model of the real system is developed and analyzed for requirements verification. This model has two phases: (1) model development and (2) model analysis. In model development, the properties of the system are listed based on the system requirements. The resulting model is analyzed to verify the expected properties and requirements. Figure 1 shows an illustration of the methodology.

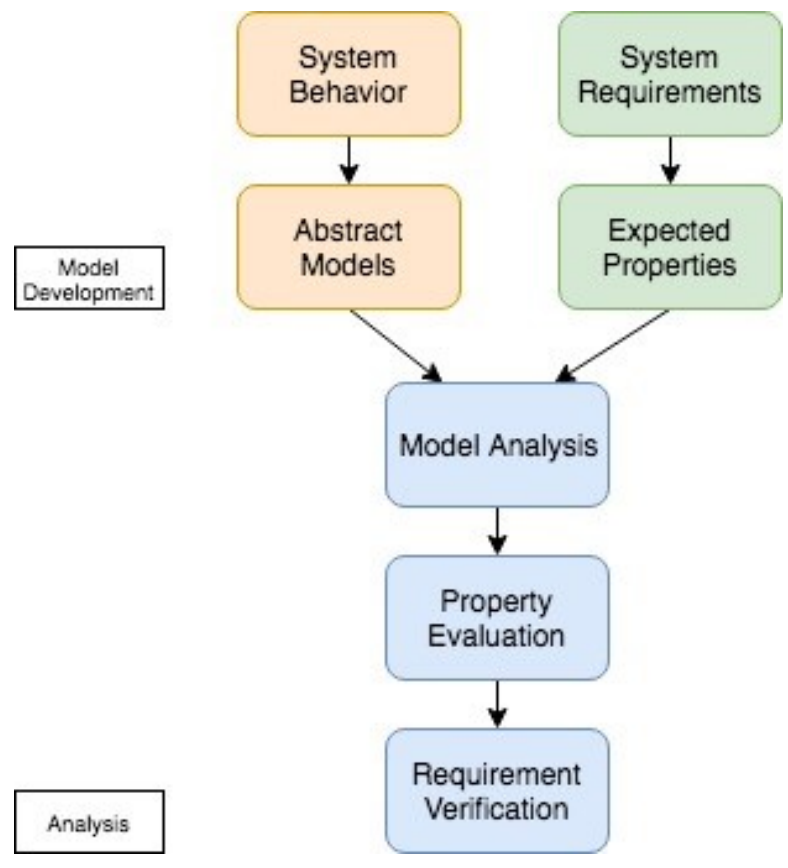

Figure 1. Model-based engineering methodology.

Hazard analysis of medical devices starts with the creation of attackers models and possible security susceptibilities in the system. In order to reinforce the security, each component is analyzed and the design is improved for avoiding vulnerabilities.

\subsection{Wearable Sensor Types}

\subsubsection{Mechanical Sensors}

Mechanical sensors detect the amount of deformation when they are strained or compressed. The changes in the shape of the sensor are converted into electrical signals. Mechanical sensors are divided into four groups: (1) piezoelectric, (2) piezoresistive, (3) capacitive and (4) iontronic [19].

\section{Piezoelectric Sensors}

Piezoelectric sensors are based on the piezoelectric effect generated by materials under external force like strain, force or pressure. The most used inorganic materials for piezoelectric sensors are zinc oxide $(\mathrm{ZnO})$, gallium nitrite $(\mathrm{GaN})$ and lead zirconate titanate (PZT), while $\mathrm{ZnO}$ nanomaterials are used to develop nanosensors. The use of inorganic materials implies a high processing cost and the use of lead, which is highly toxic for living beings. A good candidate for replacing actual inorganic piezoelectric materials is polyvinylidene fluoride (PVDF). This material has mechanical flexibility, biocompatibility, low processing cost and chemical inertness. 
A classical investigation shows the use of piezoelectric pressure sensors to develop a reliable non-invasive method of determining the peripheral arterial pressure wave velocity in humans [20]. The aim of this article is to achieve a reliable and non-intrusive way to monitor the arterial pressure in patients with arterial disease, pre- and post-surgery patients, and pre- and post-treatment patients. The measurement of elasticity of peripheral arterial blood vessels is called arterial pulse wave velocity. They measure two pulse waves at different points, one at the wrist and the second one at the brachial artery near the elbow. The pulse wave velocity is calculated by determining the pulse transit time between the points described above and the distance measured between each other. The sensors used in this application are piezoelectric devices that generate a potential difference when they are under a mechanical load. This study uses three different ways to determine the arterial pulse wave velocity: (1) foot-to-foot, which is based on the velocity of the foot of the pressure wave, (2) peak-to-peak, which is similar to the first one, the only difference being that the points of observation are two peaks of the pulse wave (proximal and distal), and (3) the coefficient of the arterial pulse at the proximal measurement position and the distal position, called cross-correlation.

In conclusion, piezoelectric sensors have high frequency response, accuracy and sensibility making them a good choice for body movement or vital sign sensors. However, some drawbacks for these kinds of sensors are poor spatial resolution and they suffer charge leaks.

\section{Piezoresistive Sensors}

These kinds of sensors use conductive materials that change their electric properties when they suffer a mechanical deformation. This is called the piezoresistive effect. Piezoresistive sensors are good detecting stress or strain. Therefore, they can be used to detect the movement of a joint in a human body. Usually, these kinds of sensors are formed by a thin film conductor on a silicone elastomer [21]. The small size allows for mounting strain sensors on the human body to detect motion like the bending of an elbow or knee. Sensors that are attached to the human body need to be flexible and comfortable to the wearer. Textile based strain sensors fulfill these requirements. Usually, textile strain sensors use a normal strain sensor embedded in textile, but new materials are conductive yarns or conductive ink. These new materials make a sensor more comfortable and water resistant.

A very recent work presents a simple and low-cost way to manufacture piezoresistive sensors using a composite of carbon black, a matrix of thermoplastic polyurethane and silver nanoparticles as sensing material [22]. This strain sensor shows more or less 18 times improvement in sensitivity at maximum strain compared with bare carbon black-based sensors. This sensor allows a high accuracy detection of finger bending, elbow flexion and wrist rotation. Sensors with higher sensitivity allow the development of accurate wearable applications to track the movement of the human body. These kinds of applications can be used to track the movement of professional athletes to increase their performance, help a patient with physical rehabilitation and track activities of an elderly person.

\section{Capacitive Sensors}

Capacitive sensors are typically composed of two parallel conductive plates separated by a space. These sensors measure changes in an electrical property called capacitance. Capacitance is the response of the conductive plates when a potential difference is applied to them. This difference creates an electric field between the two plates. Each plate has positive or negative charge and, when the polarity is changed, the charge of each plate also changes. Capacitive sensors change the polarity continuously, which generates an alternating electric current. The amount of electric current is determined by the capacitance. The capacitance varies according to the area and proximity of the plates and the material used to separate them [23].

As an example, a capacitive sensor using biocompatible ionic hydrogel films as conductive plates has been developed [24]. The flexibility of the sensor allows for measuring stretch and pressure on the human skin. Capacitive sensors are widely used, since they offer high sensitivity and low power consumption. The use of these sensors in wearable devices has some problems that have to be addressed 
properly. Parasitic noise from the human body or the environment can alter the measurements of sensors. Capacitive sensors are not a great option for developing wearable devices because they require being isolated from external noise. Nevertheless, these sensors are a great option for human interaction devices such as touch screens due to their great sensitivity and low power consumption.

\section{Iontronic Sensors}

Iontronic sensors present an improvement with respect to capacitive sensors which are highly sensitive and have a low parasitic noise. These sensors are based on a nanoscale double-layer supercapacitor. Capacitance is measured between the electrode and the electrolyte. If capacitive and iontronic sensors of a same size are compared, the second one has an electrical capacitance 1000 times higher and is immune to parasitic noise. For this reason, iontronic sensors are particularly suited for wearable devices.

In a recent paper, the authors developed an epidermal-iontronic interface (EII) [25]. This wearable interface is noise-immune and highly sensitive and adaptive. This type of sensor uses any flexible and curved surfaces with electrical conductance like the human skin as part of the device. This architecture enables sensors with high sensitivity, fast response and extended stability. The EII is able to detect internal (body) and external (environmental) stimuli. The authors successfully tested their sensor, detecting cardiovascular pulse waveforms, muscular activity tracking, respiration rate and grip pressure.

To conclude, iontronic sensors are stable and durable, making them perfect for emergency and medical applications. These types of sensors can also be applied in robotics providing robots with tactile sensors. This feature enables the development of rescue robots, which, for instance, can feel the pressure applied on a human body.

\subsubsection{Chemical Sensors}

These types of sensors respond to a change in chemical composition via an electrochemical, electrical or optical signal. The continuous exposure to biofluids can cause irreversible damage to the chemical sensor. Stability is the major challenge faced by chemical sensor designers. These types of sensors must be stable, sensitive and selective [26].

As told before, the epidermis is by nature an information barrier. This causes that a non-invasive chemical sensor has to measure biofluids secreted by the body [27]. These biofluids present more challenges because large analytes are diluted and some of these fluids are secreted in tiny amounts. Chemical sensors can be chemical-to-electrical or chemical-to-optical, each type showing several advantages and disadvantages. Chemical-to-optical sensors are ultra-low cost and simple because they do not require localized electronics or detectors. Two drawbacks of these kinds of sensors are that user interaction is required to observe or record results, and light or optic sensors should be used, which can be affected by external sources. The advantages of chemical-to-electrical sensors are that they do not require user interaction, no light sources, detector or optics are needed and they start working as soon as they are in contact with a biofluid.

The use of nanomaterials such as graphene, conducting polymers, carbon nanotubes and nanowires cause a size reduction of chemical wearable sensors and they are useful to detect a wide variety of analytes. The use of graphene has shown impressive results. Graphene is a 2D layer of carbon atoms bound in a hexagonal structure. This material shows exceptional electrical, optical, mechanical and thermal properties. Researchers experimented with graphene and proved the ability of this material detecting hydrogen peroxide $\left(\mathrm{H}_{2} \mathrm{O}_{2}\right)$, an important component for pharmaceutical analyses, heavy metals, which can lead to environmental problems, biomolecules like dopamine that in abnormal levels can indicate schizophrenia or human immunodeficiency virus, and toxic industrial chemicals, which are a health risk.

Graphene is a material with special properties that can be used in many fields. The only drawback of graphene is that it has a high production cost. Many teams are studying how to produce graphene 
in an easy and low-cost way. Chemical wearable sensors are really useful for detecting diseases or chemicals that can harm the environment. However, further research is needed to enhance wearable devices based on this material to achieve reliable and low-cost applications.

\subsubsection{Electrical Sensors}

Electrical sensors are sensitive to the electrical resistance of the skin and also measure alterations of the capacitance or the conductivity at the surface of the skin. These types of sensors requires good electrical contact with the skin to be able to produce reliable measurements. Electrical sensors use two types of contacts, wet and dry electrodes. Wet electrodes consist of a solid conductive pad and an electrolyte gel that decreases the impedance of the skin. In contrast, dry electrodes are applied in direct contact with the skin. Dry electrodes can also be non-contact dry electrodes that have an insulating layer that separates the skin from the electrode. These electrodes eliminate irritation, allergic reactions and prevent from short circuits.

In a quite recent approach, the authors developed a paper-based electrical sensor that monitors respiration [28]. The authors achieve a low-cost single-use respiration monitoring system that is used for routine and at-home monitoring. The sensor detects the humidity of the paper using the hygroscopic character. Then, it converts the changes in humidity into electrical signals. These signals are transmitted to an electronic device to be processed. When the user breathes out, the amount of water in the sensor increases, augmenting its ionic conductivity. In contrast, when the user breathes in, the level of water decreases. This device is less accurate than sensors used in laboratories but is portable and can be used outdoors.

There are other suitable applications for electrical sensors. For example, they can be used in medical applications that monitor the skin (sweating, hydration level, etc.). However, other new applications are appearing, since these sensors are suitable for augmented reality as feedback interfaces.

\subsubsection{Optical Sensors}

These sorts of devices convert light rays into electrical signals. The sensor measures the quantity of light and transforms it into a form that can be read by an instrument. Some advantages of using optical sensors are: immunity from electromagnetic interference; great sensitivity; and multiplexing capabilities. Optical sensors can be affected by external light sources and temperature variation [29].

In this regard, a research work presents a low-cost wearable gait monitor that uses optical fiber [30]. The sensor detects transmittance changes when the plastic optical fiber is bent. The wearable sensor is attached to the knee of the patient where it can measure the joint angles. The system uses a photodetector that measures the amount of light received and, after a calibration stage, it calculates the flexion angle. The authors choose fiber optic sensors because they are immune to electromagnetic interference, low weight and flexible. The results of the tests performed demonstrate that the system is sensitive enough to detect small angles. The authors believe that it can also detect hyper-extension, although further tests are needed.

The use of plastic fibers for developing wearable sensors enables the development of low cost, robust and easy-to-handle devices. Optical wearable sensors can be an important tool for rehabilitation and performance monitoring of professional athletes.

\subsection{Wearable Sensors in Healthcare}

Medicine is always searching non-intrusive methods for monitoring people health. The goal of a wearable sensor is to extract information from the body without implanting a sensor inside the body. This means that sensors must be attached to the epidermis in most cases. The epidermis by nature acts as a barrier. The epidermis is the first line of defense of the immune system also protecting the body from the loss of water, nutrients, and solutes. For this reason, the epidermis presents the first challenge to the wearable sensors to be able to obtain information from the body [27]. 
Wearable sensors allow for monitoring vital signs of a patient [31]. This amount of information helps doctors to detect diseases with more confidence and also helps professional athletes to improve their performance. The health of a person can be measured with a collection of physiological aspects. Roles of each aspect and the interaction between them help doctors to identify disorders. These indicators are grouped into (1) body motions, (2) vital signs and (3) metabolism parameters [19].

Body motion is usually measured using strain and pressure sensors. Possible applications for these kinds of sensors are rehabilitation, gesture identification, gait detection or expression identification [32,33]. As an example, please consider a smartwatch application named ADAM (Advanced Daily Activity Monitor) [34]. ADAM is only one node of a BAN solution. This solution monitors elderly people daily activities. The BAN application contains algorithms for fall detection, gait alteration detection and activity recognition. Very recently, an IoT-based health monitoring system uses electrocardiogram sensor, body temperature sensor, pulse rate, and oxygen saturation sensor [35].

Body motion detection is fundamental in some healthcare-related areas like sports. BANs applied to sports are used to help people for improving their progress, or professional athletes for finding defects in their performance. They are also widely used in rehabilitation of injuries caused by sport $[36,37]$. The measurements are typically focused on data of movements performed by the user, which are acquired by sensors such as accelerometers, gyroscopes, magnetometers, pressure gauges, etc. Data provided by these sensors are analyzed to assess, for instance, the physical ability of users, determining if a movement is properly executed, to know the speed reached, the amount of calories and fat burned, your heart rate, or the distance traveled in the exercise [4]. Moreover, BANs are also utilized in virtual and augmented reality to detect body movements or hand gestures to interact with objects within a virtual environment [38]. Another approach presents a wearable composed of three-axis absolute orientation sensor, accelerometer and gyroscope [39].

Vital signs like heart rate, skin temperature or respiration use temperature, strain, pressure and humidity devices. These sensors are applied for the detection of cardiovascular diseases, heart failures, apnea and emotional control, or wound healing and physiological status. An example of a BAN system that monitors vital signs of patients that suffer from hyperthyreosis, sleep apnea, or sudden infant death syndrome has been proposed [40]. The application uses a wearable digital respiratory sensor and a breathing pattern monitor that helps to identify an abnormal breathing. The tests have shown that the application detects abnormal respiratory effectively. However, the system has only been tested eight times with six different subjects. More tests are needed to verify the accuracy of this solution. A couple of recent papers have introduced the estimation of mental distress from photoplethysmography [41] and electrodermal activity [42].

In case of the metabolism, chemical sensors are used in sweating areas and cavities to evaluate the body status by the concentration of alcohol, $\mathrm{pH}$, lactic acid and electrolytes. These sensors can be combined with multiple configurations depending on the application. For example, gait detector, temperature, heart rate, and respiration sensors are used together to improve the performance of a professional athlete or alert about heart failures from patients with heart diseases.

Lastly, let us highlight that wearable sensors capturing physiological data are used for applications beyond healthcare as such. For example, a biometric authentication system using electrocardiograms provided by a single mobile chip has been proposed [43]. The authentication follows the next scheme: (1) get the electrocardiograms from the mobile sensor, (2) apply a canceling noise filter to the data, (3) extract the features of the heartbeats, and (4) authenticate the user. The research team tests the solution in 175 subjects. The result of those tests showed that around $5 \%$ of authentication using a single heartbeat experiments failed and around $2 \%$ of authentication with 15 seconds of heartbeat data tests failed. With these results, the authentication system proves to be a viable solution.

Wearable sensors are divided into four groups [27]: mechanical, chemical, electrical, and optical. The use of each group depends on the characteristics of the application. Each type of sensor has its own requirements. In the case of chemical sensors, they must be able to detect chemical concentration rapidly with short responses. The major issue with chemical sensors is their stability. The continuous 
exposure to biofluids can lead to false positives. Mechanical sensors can suffer from deformation, mechanical degradation or failures by continuous use. Usually, optical and electrical sensors are more reliable and robust as there are fewer moving parts. Each type of sensor presents setbacks. For this reason, teams around the world are investigating ways for improving the stability of these sensors. The following subsections describe each type of sensor, focusing on the advantages and weaknesses of every technology.

\section{Data Link Layer Properties}

\subsection{Properties According to IEEE 802.15.16 Standard}

The data link layer includes the medium access control (MAC) sublayer, which manages access and contention to the channel. Data are transmitted using time-domain multiplexing. The hub splits the time axis into a series of super-frames. The MAC specification supports QoS, medical implant communication service (MICS), emergency communications, interference mitigation, and macroscopic and microscopic power management. IEEE 802.15.6 has the following operation modes: beacon mode with beacon period super-frame boundaries; non-beacon mode with super-frame boundaries; and non-beacon mode without super-frame boundaries.

A survey on different MAC protocols and their comparison for radio BANs can be consulted [44]. In a study, the focus was put on the integration of fuzzy-logic based routing with a time-division multiple access MAC protocol [45]. In addition, the improvement of the IEEE 802.15.4 MAC protocol has been addressed in [46]. The authors present the so-called BANMAC, which is a MAC protocol capable of monitoring and predicting channel fluctuations and scheduling transmissions, improving packet loss and reducing power consumption.

\subsection{Sustainability}

Awareness of the environment is increasing in recent years. Big and small companies are developing environmental friendly products. Computing systems contribute to the environment with two aspects: (1) energy consumption and (2) use of renewable materials.

Energy efficiency is an essential feature in BAN applications. Batteries used in these applications should not be replaced in many years. This goal can be achieved by using strategies for optimal energy consumption. Usually, communication is the action that drains more energy. Energy consumption can be optimized using energy efficient medium access protocols. These protocols can turn off the transmitter when it is not required. Another technique is allotting slots dynamically. If a transition fails, allocated slots prevent waste of energy and time.

Energy for powering nodes in a BAN system can come from different sources. These sources can be solar power, pressure variation, vibrations, air flow, human power and temperature. A human produces body heat that is equivalent to a power density of $20 \mathrm{~mW} / \mathrm{cm}^{2}$ [47]. This energy can be gathered by thermoelectric generators. These generators can store energy in a battery which powers the node. The use of human body in BAN applications can produce more energy efficient solutions. It is also possible to use the human body as a communication channel, and some studies are being developed to improve this technology.

There are different strategies for reducing the nodes' energy requirements:

- Workload management ensures that the tasks are balanced among all nodes of the system. This prevents overloading states causing a higher power consumption. It is also possible to delay a work (not affecting the requirements) until the energy source is ready.

- Power management helps to reduce energy consumption. This involves the administration of sleep and idle modes in the processor when it is not required. The same process can be applied to communication devices.

- The energy demanded is typically due to non-computing processes. An efficient management of these processes can reduce significantly power requirements of the application. 
Preserving the environment is also a great concern. A good design is mandatory to reduce the pollution in BAN applications. Two strategies are used for protecting the environment when developing BAN solutions: (1) using eco-friendly materials that are recycled with a minimum impact and (2) designing durable nodes, avoiding partial or total replacement, thus reducing waste.

The use of green energy for powering a BAN application may also lead to problems. Usually, the available energy is not sufficient to operate the devices. This problem is caused by unknown load characteristics. Sustainability research proposes three ways to overcome this problem [15]:

1. The first one is to improve the efficiency of energy harvesting from the environment,

2. The second one is to adjust the energy source to the duty cycling of the devices,

3. Finally, the use of energy-efficient computing.

The aim of these techniques is to achieve an energy balance. A system reaches energy neutrality when it consumes the same energy harvested. Harvesting theory is an analytic method that is used to solve this problem. This theory uses a model-based approach and stochastic models of the harvesting source and the device. The harvesting theory can be used to find the best duty cycle of the device while maintaining energy balance. Energy consideration is still a hot topic in BANs. A recent paper intends to find the optimal QoS and energy consumption to increase profits [48].

A proper design is fundamental to achieve this balance. The design methodology has three phases: (1) profiling phase, (2) modeling phase, and (3) analysis and design phase. MBE has similar phases and both methodologies can be implemented at the same time. Both methodologies require gathering the requirements for modeling the components interactions and the evaluation of the expected results. MBE focuses on the safety and the design methodology in the efficiency of the solution. In the profiling phase, trials are executed to obtain workload data from the processor, radio power, and thermal characteristics. Power consumption of devices is evaluated using different power-management strategies. In this phase, the sources are evaluated to know the amount of energy that can gather as well.

The information obtained during the profiling phase is used in the modeling phase. The goal of this phase is to model the behavior of the BAN system and the human body. The last phase consists of two subphases: (1) analysis phase and (2) design phase. The analysis phase selects a model from the modeling phase and a set of requirements. These requirements are used as inputs and outputs of the model. The design phase uses the analysis phase to determine the best strategy for a sustainable BAN design. This phase finds the best solution faster than a brute-force search.

Sustainability of healthcare BAN-based applications can benefit from other groundbreaking domains like Industry 4.0. Indeed, BANs are being introduced to assist and monitor workers. A recent article seeks to walk towards the social sustainability of the industry [49]. An intelligent band has been tested in an emulated industrial scenario to correct a worker's tasks to avoid possible injuries [50].

\section{Network Layer Components, Configuration and Middleware Layer}

As mentioned before, a BAN is a network of nodes with sensors and actuators that communicate with each other. These nodes are worn on the body and store environmental, activity and/or physiological information [15]. BAN nodes are commonly classified into two broad categories:

- Peripheral nodes: Devices with a low processing platform. Peripheral nodes have sensors to monitor their environment and communication interfaces to transfer and receive information.

- Central nodes: Devices with enhanced processing and communication capabilities. Central nodes are in charge of gathering the information of the sensor nodes and make the data available (usually through the cloud).

A good architecture is vital for developing wearable applications, especially for emergency and medical systems where a human life depends on a correct acquisition of information in some cases. Regarding the hardware of BAN nodes, there are multiple configurations. The IEEE 802.15 task group 6 (IEEE TG6) [51] suggests the following types of nodes: 
- A low-power microcontroller attached with multiple sensors that monitor the environment,

- Nodes for medical purposes,

- Devices that collect or aggregate information,

- Nodes that control the administration of medication like an infusion-pump controller,

- Devices acting like a gateway or access point.

The selection of hardware for a BAN application depends on the requirements of the system to be developed. This is the reason why it is highly important to understand the problem at hand in order to choose the right components to build the whole system.

As mentioned before, generally, a BAN is composed of two different nodes: (1) peripheral nodes with a low-power microcontroller, and (2) central nodes that manage the information generated by the peripheral nodes. The network topology means the arrangement of nodes in a BAN, describing the communication between nodes. There are several options to connect nodes in a BAN. The IEEE TG6 suggests three types of network topologies, as shown in Figure 2. In Figure 2a, representing a star topology, each peripheral node has a direct connection to the central node. Another recommended topology is mesh topology, as depicted in Figure 2b. In a mesh topology, not all peripheral nodes have a direct connection to the central node. Lastly, Figure $2 c$ shows a combination of the previous topologies, which is a hybrid topology.

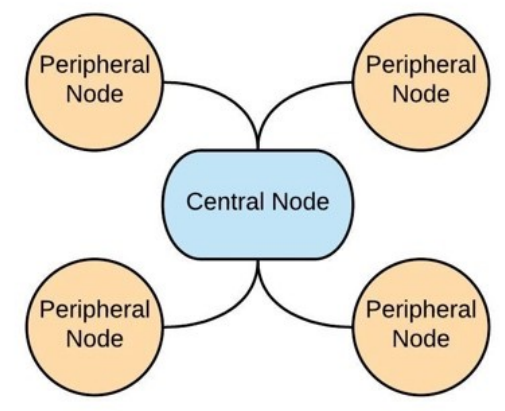

(a)

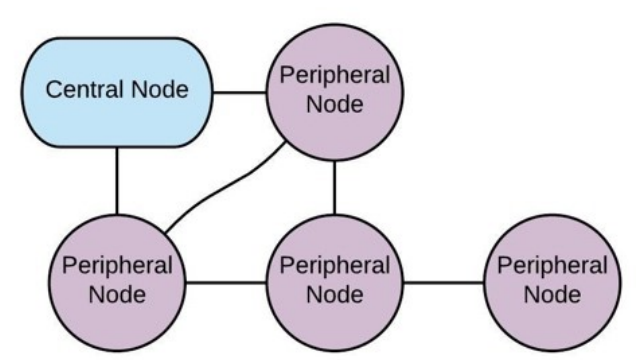

(b)

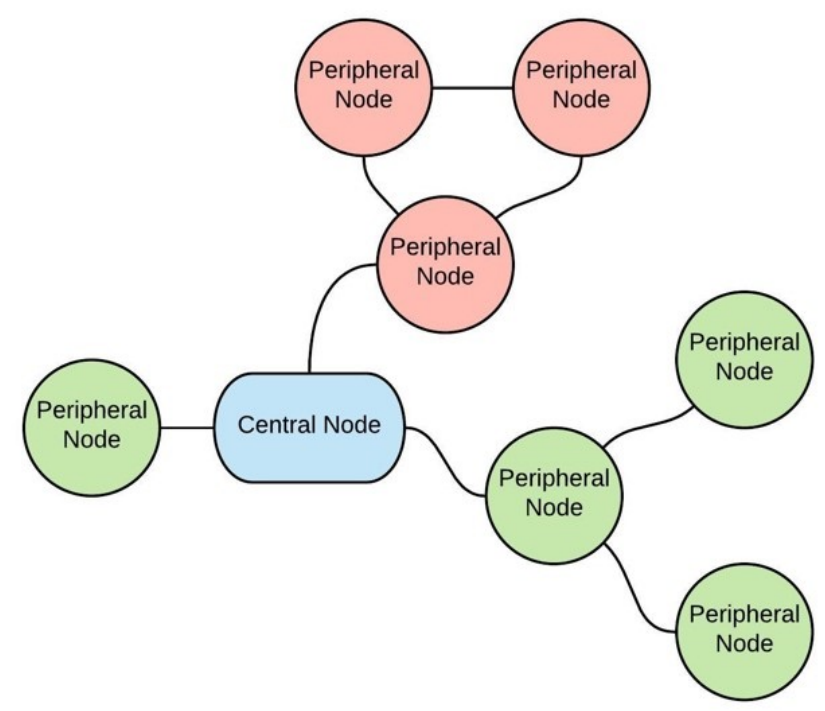

(c)

Figure 2. Body area network topologies: (a) star topology; (b) mesh topology; and (c) hybrid topology.

In addition to the network layer configuration, the middleware layer is also relevant in order to provide an abstraction mechanism making the system able to interact with different components in an easy manner. There are some articles that tackle this issue, providing specific platforms to enable the use of diverse devices as coordinators of the body sensor network and body-side of the 
cloud platform [52,53], treating interoperability issues between different IoT platforms oriented to healthcare services, and performing a fusion of sensor data to the detection of emotions $[54,55]$. Regarding this topic, data fusion in body sensor networks contributes with interesting articles [56]. The authors performed a survey about emotion recognition and general health articles. Finally, in another example of the use of body sensors in healthcare [57], an electrocardiogram analysis is performed to automatically detect the cardiac defence response to understand the patient's mental and health status.

\section{Cross-Layer Properties}

\subsection{Privacy and Security}

In the majority of cases, BAN applications manage sensitive information. This information must be protected from unauthorized access or modification. With the aim of protecting sensitive data, basic requirements must be satisfied in a system:

- $\quad$ The information generated by the solution needs to be accurate and without alterations.

- Sensitive data can only be accessed by authorized entities.

- The system must know the identities of all entities that access it.

- Entities only have access to the necessary information.

- The information generated by the application needs to be available when required.

A possible violation of security is a threat. An attack is when a threat is executed by entities called attackers. Threats can be grouped into three categories:

1. When the attacker is observing the communication of the system with another authorized entity and intercepts the transmitted data without interfering. This attack is called eavesdropping.

2. An attack made by an entity that has a valid part of the system by modifying, deleting or adding information is called spoofing.

3. Denial of service attack is when an attacker inhibits the availability of the system. This attack can be done by blocking the communication channel or by physical damage to the hardware.

The IEEE 802.15.6 standard proposes three different levels of security:

1. Unsecure: At this level, none of the basic security requirements are satisfied. This is an unsecured communication.

2. Authentication: A system using this level of security uses authentication but not encryption for transmitting information.

3. Authentication and encryption: This level uses authentication and encryption for communication. This is the most secure level.

In medical applications, the security of patient records should be considered in all development phases. Medical records are sensitive in nature and must be protected from unauthorized access. Unapproved access to patient records can lead to many problems, such as:

- Incorrect diagnosis due to an alteration of medical records,

- False alarms,

- Elimination of real alarms,

- Patients can suffer economic and social discrimination.

On the one hand, BAN applications are continuously gathering and storing sensitive information, and this aspect can lead to security breaches. Developers must ensure that the information generated by BAN systems is secured. Adding security causes an increase in complexity, which could make the 
system difficult to develop and debug; difficult to use for caregivers and patients; expensive, and also computationally. However, a good design in the development process can solve or mitigate many of the issues previously mentioned.

In this regard, a lightweight anomaly detection framework for BAN applications has been described [58]. The framework is based on four algorithms: discrete Haar Wavelet transform, non-seasonal Holt-Winters, Hampel filter, and Boxplot. Through these algorithms, the framework is capable of detecting faulty or malicious injected measurements in healthcare solutions. The framework was designed to be used in devices with low computational complexity and storage requirements. Another example is a design in healthcare monitoring systems for a proactive reliable data transmission mechanism with resilience requirements in a many-to-one stream model [59].

\subsection{Common Security Solutions}

A secure communication is composed of two parts: trust establishment and communication. The first part means that the devices involved in the communication ensure their legitimacy. For this purpose, cryptography is widely used. In the case of symmetric keys, each entity has a copy of the key. This key is used to encrypt and decrypt a message and, therefore, to establish a secure communication. Another method is the use of asymmetric keys, where each device has a pair of keys: one key for encryption and the other for decryption. Asymmetric key cryptosystems consume more resources than systems that use only symmetric keys.

In the case of BAN systems, they are composed of several layers. The importance of securing each layer is listed below:

- The exchange of information between a BAN device and a smartphone must be secured because sensitive data are transmitted. Cryptography techniques are commonly used to secure this communication.

- Smartphones transmit private information to the cloud in a server-client manner using wireless internet access. Wifi protected access (WPA) or WPA2 encryption are used to protect the exchange of information.

- All places that store sensitive information have to be secured in order to ensure privacy. Secret sharing and code erasing have been proposed in the case of securing sensor storage.

- The access of sensitive information has to be performed by authorized people. This ensures the patients' privacy. For controlling the access of sensitive data access-control policies, role-based access control and criticality-aware access rules are commonly used.

There is a security protocol for BAN applications called physiological-signal-based key agreement (PSKA) that combines signal processing with cryptographic primitives. This protocol allows automatic key agreement between devices using physiological signals from the human body. This protocol consists of five main phases:

1. Devices involved in the information exchange agree beforehand on the use of a physiological signal.

2. The physiological signal is processed and transformed into a binary string.

3. The sender device uses an arbitrary key to encrypt the message.

4. The sender hides the key using the binary string obtained from the physiological signal. Then, it sends the encrypted message and the hidden key in the same package.

5. The receiver uses the local binary string to obtain the key. After that, the device verifies the key decryption/checking the integrity of data received.

After the process presented above, subsequent data exchange takes place without using a physiological signal. This protocol uses the observation of physiological signals which are unique at any time as a security method. Therefore, an external device that does not have contact with the patient 
cannot exchange information with other devices. PSKA protocol does not require any additional key to secure a communication between devices. This protocol also allows adding, removing or moving devices without a significant change in the network.

PSKA protocol is an innovative way of ensuring a secure communication in BAN applications, but it has its challenges. The first challenge that has to be solved is to decide which physiological signal is used to secure the exchange of information. The human body produces a countless number of signals and PSKA decides which one is vital to use. The chosen signal has to be processed and this can lead to several issues explained below:

- The physiological signal chosen has to meet the following properties: length and randomness, low latency, distinctiveness and temporal variance.

- Physiological signals by nature are time-variant and the key-unhiding process needs synchronization between the receiver and the sender in order to be used.

- Another aspect to be taken into account is the topographic specificity of the human body. Physiological signals apparently have similar trends but not exactly the same values when measured at different parts of the body.

- A key aspect of physiological signals is that they are time-variant and unpredictable. This prevents external sources from knowing future values if the current value is known. This aspect also presents a challenge, as the communicating devices must be synchronized to perform a secure exchange of data.

The processing of physiological signals can be performed in two ways. In the first one, time-domain-analysis signal values are transformed into binary streams and concatenated. The problem with this approach is that devices must be synchronized. The second one is frequency-domain analysis, which produces similar values even if devices are not synchronized. However, this method requires mathematical transformations that can be expensive, especially in resource-constrained devices.

An example of security applied to medical data transmission is a recent proposal [60], where the authors designed a framework for healthcare able to improve the security and privacy through the use of homomorphic encryption. In addition, some researchers have proposed a four-tier architecture for health monitoring systems [61]. They identify the security requirements in each layer and review the literature on improving WBANs security and privacy. Moreover, apart from security aspects, there are studies related to the mixture of protocols using cross-layer bridges to address congestion issues in sensor networks [62].

\section{Current Challenges}

The development of new sensors, communication channels, wireless networks, and secure and safe smart integrated components for cyberphysical systems is an area of great interest. According to the European Commission [63], this is a priority in the Horizon 2020 research and innovation programme, where the reinforcement of the integration of embedded components and cyberphysical systems is crucial to achieve interconnected and collaborating spaces. As mentioned before, these kinds of systems need to comply with several requirements like security and safety, low power consumption and size, usability and adaptability [64].

Body area networks are still a relatively new technology with problems and limitations that must be solved in order to reach the standards required for extensive use. Many research teams around the world are working to look for solutions to turn BAN products in effective deployments. The principal challenges that BANs face are: signal propagation, interference and human-computer interaction [47].

BAN nodes, on or within the human body, are affected by several communication issues. The strength of the signal varies depending on the location and orientation of the nodes. Some researchers are studying ways to transmit information among devices using the human body as a means of transmission [12]. 
Interference becomes a serious problem when there are several devices or BANs emitting simultaneously. This causes increased packet loss due to transmission channel saturation. Researchers are developing ways to deal with interference. One is the use of biometrics (glucose level, blood pressure, heart rate) to identify a BAN user. Another is the use of multiple channels, where each user could have its own communication channel. The use of multiple channels enhances packet delivery, thus greatly preventing packet loss.

Any technological solution should have a user-friendly interface to show the information status or to receive commands. In the case of BAN systems, usability requirements are high. Users of this type of devices are usually unfamiliar with the use of this technology. To avoid this difficulty, some researchers are manufacturing T-shirts with sensor tissue that can monitor the wearer by themselves.

\section{Conclusions}

This work has presented a brief state of the art of the body area network technology, with a special emphasis on BAN applications in the healthcare domain. The objective of this paper has been to shortly review the architecture, network topology, energy sources, applications and main challenges for these systems.

In this regard, the IEEE 802.15 standard provides guidelines for BAN applications and defines different layers that these types of solutions must follow. Security is a really important feature in BAN products as the information generated by the applications must be accessible only to people who are supposed to have access to the data provided. Sustainability is another significant factor in developing BAN solutions as well. A good design leads to a durable and eco-friendly product.

Different types of wearable sensors have also been presented, highlighting the advantages and weaknesses of each one. Studies from teams around the world working on different wearable applications have been introduced. These studies show the great potential for innovative systems that use these kinds of sensors. In conclusion, we have presented a survey on the state of the art of the latest BAN solutions in healthcare as provided by the leading researchers. Throughout this article, many publications have been referenced with the aim of showing the manifold benefits that BANs bring to the area.

Regarding future studies, it is our intention to investigate other topics related to BAN solutions apart from the healthcare arena. This will help to obtain a wider view of this technology in other important fields of research.

Author Contributions: Conceptualisation, A.P., A.F.-C. and N.C.; methodology, D.V. and L.R.-S.; investigation, L.R.-S. and T.O.; writing, D.V., L.R.-S. and T.O.; funding acquisition, A.P. and A.F.-C.

Acknowledgments: This work was partially supported by FCT - Fundação para a Ciência e Tecnologia, I.P., under projects UID/CEC/04524/2016 and UID/CEC/04524/2019. This work was partially supported by Spanish Ministerio de Economía, Industria y Competitividad, Agencia Estatal de Investigación (AEI)/European Regional Development Fund (FEDER, UE) under DPI2016-80894-R grant.

Conflicts of Interest: The authors declare no conflict of interest.

\section{Abbreviations}

The following abbreviations are used in this manuscript:

$\begin{array}{ll}\text { BAN } & \text { Body area network } \\ \text { IEEE TG6 } & \text { IEEE 802.15 Task Group 6 } \\ \text { IoT } & \text { Internet of Things } \\ \text { HBC-PHY layer } & \text { Human body communication physical layer } \\ \text { MAC layer } & \text { Medium access control layer } \\ \text { NB-PHY layer } & \text { Narrow band physical layer } \\ \text { PHY layer } & \text { Physical layer } \\ \text { PSKA } & \text { Physiological-signal-based key agreement } \\ \text { QoS } & \text { Quality of service }\end{array}$




$\begin{array}{ll}\text { UWB-PHY layer } & \text { Ultra-wide band physical layer } \\ \text { WBAN } & \text { Wireless body area network } \\ \text { WPA } & \text { Wifi protected access }\end{array}$

\section{References}

1. United Nations, Department of Economic and Social Affairs, Population Division. World Population Prospects: The 2017 Revision-Key Findings and Advance Tables. 2017. Available online: https:/ / reliefweb. int/report/world/world-population-prospects-2017-revision-key-findings-and-advance-tables (accessed on 7 August 2019).

2. World Health Organization. The World Health Report 2002: Reducing Risks, Promoting Healthy Life. 2002. Available online: https:/ /apps.who.int/iris/handle/10665/67454 (accessed on 7 August 2019).

3. Reichman, A. Body area networks: Applications, architectures and challenges. IFMBE Proc. 2009, $25,4043$.

4. Asare, D.A.K. Body Area Network Standardization, Analysis and Application. Bachelor's Thesis, Savonia University of Applied Sciences, Kuopio, Finland, 2014.

5. Latré, B.; Braem, B.; Moerman, I.; Blondia, C.; Demeester, P. A survey on wireless body area networks. Wirel. Netw. 2011, 17, 1-18. [CrossRef]

6. Chen, M.; Gonzalez, S.; Vasilakos, A.; Cao, H.; Leung, V.C.M. Body area networks: A survey. Mobile Netw. Appl. 2011, 16, 171-193. [CrossRef]

7. Seyedi, M.; Kibret, B.; Lai, D.T.H.; Faulkner, M. A survey on intrabody communications for body area network applications. IEEE Trans. Biomed. Eng. 2013, 60, 2067-2079. [CrossRef] [PubMed]

8. Smith, D.B.; Miniutti, D.; Lamahewa, T.A.; Hanlen, L.W. Propagation models for body-area networks: A survey and new outlook. IEEE Antenn. Propag. M 2013, 55, 97-117. [CrossRef]

9. Cavallari, R.; Martelli, F.; Rosini, R.; Buratti, C.; Verdone, R. A survey on wireless body area networks: Technologies and design challenges. IEEE Commun. Surv. Tutor. 2014, 16, 1635-1657. [CrossRef]

10. Kompara, M.; Hölbl, M. Survey on security in intra-body area network communication. Ad Hoc Netw. 2018, 70, 23-43. [CrossRef]

11. Salayma, M.; Al-Dubai, A.; Romdhani, I.; Nasser, Y. Wireless body area network (WBAN): A survey on reliability, fault tolerance, and technologies coexistence. ACM Comput. Surv. 2017, 50, 3. [CrossRef]

12. Dhanvijay, M.M.; Patil, S.C. Internet of Things: A survey of enabling technologies in healthcare and its applications. Comput. Netw. 2019, 153, 113-131. [CrossRef]

13. Yessad, N.; Omar, M.; Tari, A.; Bouabdallah, A. QoS-based routing in wireless body area networks: A survey and taxonomy. Computing 2018, 100, 245-275. [CrossRef]

14. Punj, R.; Kumar, R. Technological aspects of WBANs for health monitoring: A comprehensive review. Wirel. Netw. 2019, 25, 1125-1157. [CrossRef]

15. Gupta, S.K.; Mukherjee, T.; Venkatasubramanian, K.K. Body Area Networks: Safety, Security, and Sustainability; Cambridge University Press: Cambridge, UK, 2013.

16. Al Masud, S.M.R. QoS taxonomy towards wireless body area network solutions. Int. J. Appl. Innov. Eng. Manag. 2013, 2, 221-234.

17. Kwak, K.S.; Ullah, S.; Ullah, N. An overview of IEEE 802.15.6 standard. In Proceedings of the 2010 3rd International Symposium on Applied Sciences in Biomedical and Communication Technologies, Rome, Italy, 7-10 November 2010; pp. 1-6.

18. International Organization for Standardization. IEC 60601-1-11:2015. Medical Electrical Equipment-Part 1-11: General Requirements for Basic Safety and Essential Performance-Collateral Standard: Requirements for Medical Electrical Equipment and Medical Electrical Systems Used in the Home Healthcare Environment. Available online: https:/ / www.iso.org/standard/65529.html (accessed on 7 August 2019).

19. Liu, Y.; Wang, H.; Zhao, W.; Zhang, M.; Qin, H.; Xie, Y. Flexible, stretchable sensors for wearable health monitoring: Sensing mechanisms, materials, fabrication strategies and features. Sensors 2018, 18, 645. [CrossRef] [PubMed]

20. McLaughlin, J.; McNeill, M.; Braun, B.; McCormack, P.D. Piezoelectric sensor determination of arterial pulse wave velocity. Physiol. Meas. 2003, 24, 693. [CrossRef] [PubMed]

21. Farooq, M.; Sazonov, E. Strain sensors in wearable devices. In Wearable Electronics Sensors_ For Safe and Healthy Living; Springer: Cham, Switzerland, 2015; pp. 221-239, ISBN 978-3-319-18191-2. 
22. Zhang, W.; Liu, Q.; Chen, P. Flexible strain sensor based on carbon black/silver nanoparticles composite for human motion detection. Materials 2018, 11, 1836. [CrossRef] [PubMed]

23. Lion Precision. Capacitive Sensor Operation and Optimization (How Capacitive Sensors Work and How to Use Them Effectively). Capacitive Sensor TechNote LT03-0020. 2009. Available online: http:/ /www. lionprecision.com/tech-library/technotes/cap-0020-sensor-theory.html (accessed on 7 August 2019).

24. Sun, J.-Y.; Zhao, X.; Illeperuma, W.R.K.; Chaudhuri, O.; Oh, K.H.; Mooney, D.J.; Vlassak, J.J.; Suo, Z. Highly stretchable and tough hydrogels. Nature 2012, 489, 133-136. [CrossRef] [PubMed]

25. Zhu, Z.; Li, R.; Pan, T. Imperceptible epidermal-iontronic interface for wearable sensing. Adv. Mater. 2018, 30, 1705122. [CrossRef] [PubMed]

26. Ammu, S. Graphene based chemical sensors. Sci. Lett. 2015, 4, 162.

27. Heikenfeld, J.; Jajack, A.; Rogers, J.; Gutruf, P.; Tian, L.; Pan, T.; Li, R.; Khine, M.; Kim, J.; Wang, J. Wearable sensors: Modalities, challenges, and prospects. Lab Chip 2018, 18, 217-248. [CrossRef]

28. Guder, F.; Ainla, A.; Redston, J.; Mosadegh, B.; Glavan, A.; Martin, T.J.; Whitesides, G.M. Paper-based electrical respiration sensor. Angew. Chem. Int. Ed. 2016, 55, 5727-5732. [CrossRef]

29. Ahuja, D.; Parande, D. Optical sensors and their applications. Int. J. Sci. Res. Rev. 2012, 1, 60-68.

30. Bilro, L.; Pinto, J.L.; Oliveira, J.; Nogueira, R. Gait monitoring with a wearable plastic optical sensor. IEEE Sens. 2008, 787-790.

31. Angelidis, P. Personalised physical exercise regime for chronic patients through a wearable ICT platform. Int. J. Electron. Health 2010, 5, 355-70. [CrossRef] [PubMed]

32. Castillo, J.C.; Carneiro, D.; Serrano-Cuerda, J.; Novais, P.; Fernández-Caballero, A.; Neves, J. A multi-modal approach for activity classification and fall detection. Int. J. Syst. Sci. 2014, 45, 810-824. [CrossRef]

33. Sokolova, M.V.; Serrano-Cuerda, J.; Castillo, J.C.; Fernández-Caballero, A. A fuzzy model for human fall detection in infrared video. J. Intell. Fuzzy Syst. 2013, 24, 215-228.

34. Genovese, V.; Mannini, A.; Sabatini, A.M. A smartwatch step counter for slow and intermittent ambulation. IEEE Access 2017, 5, 13028-13037. [CrossRef]

35. Islam, M.S.; Islam, M.T.; Almutairi, A.F.; Beng, G.K.; Misran, N.; Amin, N. Monitoring of the human body signal through the Internet of Things (IoT) based LoRa wireless network system. Appl. Sci. 2019, 9, 1884. [CrossRef]

36. Jovanov, E.; Milenkovic, A.; Otto, C.; de Groen, P. A wireless body area network of intelligent motion sensors for computer assisted physical rehabilitation. J. Neuroeng. Rehabil. 2005, 2, 6. [CrossRef]

37. Waghmare, A.M.; Ghare, P.H. Performance improvement for multi-BAN routing protocol for soccer player monitoring and study of factors contributing towards efficiency of the protocol. In Proceedings of the 2018 9th International Conference on Computing, Communication and Networking Technologies, Bengaluru, India, 10-12 July 2018.

38. Barrie, P.; Komninos, A.; Mandrychenko, O. A pervasive gesture-driven augmented reality prototype using wireless sensor body area networks. In Proceedings of the 6th International Conference on Mobile Technology, Application \& Systems, Nice, France, 2-4 September 2009; p. 61.

39. Laibowitz, M.; Samanta, V.; Ali, S.; Azuma, R. Chamber of mirrors: A socially activated game exploits pervasive technology. IEEE Pervasive Comput. 2012, 11, 38-45. [CrossRef]

40. Liu, L.; Shah, S.A.; Zhao, G.; Yang, X. Respiration symptoms monitoring in body area networks. Appl. Sci. 2018, 8, 568. [CrossRef]

41. Zangróniz, R.; Martínez-Rodrigo, A.; López, M.T.; Pastor, J.M.; Fernández-Caballero, A. Estimation of mental distress from photoplethysmography. Appl. Sci. 2018, 8, 69. [CrossRef]

42. Zangróniz, R.; Martínez-Rodrigo, A.; Pastor, J.M.; López, M.T.; Fernández-Caballero, A. Electrodermal activity sensor for classification of calm/distress condition. Sensors 2017, 17, 232. [CrossRef] [PubMed]

43. Choi, H.S.; Lee, B.; Yoon, S. Biometric authentication using noisy electrocardiograms acquired by mobile sensors. IEEE Access 2016, 4, 1266-1273. [CrossRef]

44. Bhandari, S.; Moh, S. A survey of MAC protocols for cognitive radio body area networks. Sensors 2015, 15, 9189-9209. [CrossRef] [PubMed]

45. Ortiz, A.M.; Royo, F.; Olivares, T.; Orozco-Barbosa, L.; Castillo, J.C.; Fernández-Caballero, A. Protocol integration for intelligent monitoring applications in wireless sensor networks. In Foundations on Natural and Artificial Computation; Springer: Berlin, Germany, 2011; pp. 511-520. 
46. Prabh, K.S.; Royo, F.; Tennina, S.; Olivares, T. A MAC protocol for reliable communication in low power body area networks. J. Syst. Arch. 2016, 66-67, 1-13. [CrossRef]

47. Johny, B.; Anpalagan, A. Body area sensor networks: Requirements, operations, and challenges. IEEE Potentials 2014, 33, 21-25. [CrossRef]

48. Samanta, A.; Li, Y. Distributed pricing policy for cloud-assisted body-to-body networks with optimal QoS and energy considerations. IEEE Trans. Serv. Comput. 2018. [CrossRef]

49. Roda-Sanchez, L.; Garrido-Hidalgo, C.; Hortelano, D.; Olivares, T.; Ruiz, M.C. OperaBLE: An IoT-based wearable to improve efficiency and smart worker care services in Industry 4.0. J. Sens. 2018, 2018, 6272793. [CrossRef]

50. Garrido-Hidalgo, C.; Hortelano, D.; Roda-Sanchez, L.; Olivares, T.; Ruiz, M.C.; Lopez, V. IoT heterogeneous mesh network deployment for human-in-the-loop challenges towards a social and sustainable Industry 4.0. IEEE Access 2018, 6, 28417-28437. [CrossRef]

51. IEEE 802.15 WPAN Task Group 6 (TG6). Body Area Networks. Available online: http://ieee802.org/15/ pub/TG6.html (accessed on 7 August 2019).

52. Fortino, G.; Giannantonio, R.; Gravina, R.; Kuryloski, P.; Jafari, K. Enabling effective programming and flexible management of efficient body sensor network applications. IEEE Trans. Human-Mach. Syst. 2013, 43, 115-133. [CrossRef]

53. Fortino, G.; Parisi, D.; Pirrone, V.; Di Fatta, G. BodyCloud: A SAAS approach for community body sensor networks. Future Gener. Comput. Syst. 2014, 35, 62-79. [CrossRef]

54. Pace, P.; Aloi, G.; Caliciuri, G.; Gravina, R.; Savaglio, C.; Fortino, G.; Ibanez-Sanchez, G.; Fides-Valero, A.; Bayo-Monton, J.; Uberti, M.; et al. INTER-Health: An interoperable IoT solution for active and assisted living healthcare services. IEEE World Forum Int. Things (WF-IoT) 2019, 81-86.

55. Fortino, G.; Galzarano, S.; Gravina, R.; Li, W. A framework for collaborative computing and multi-sensor data fusion in body sensor networks. Inf. Fusion 2015, 22, 50-70. [CrossRef]

56. Gravina, R.; Alinia, P.; Ghasemzadeh, H.; Fortino, G. Multi-sensor fusion in body sensor networks: State-of-the-art and research challenges. Inf. Fusion 2017, 35, 68-80. [CrossRef]

57. Gravina, R.; Fortino, G. Automatic methods for the detection of accelerative cardiac defense response. IEEE Trans. Affect. Comput. 2016, 7 286-298. [CrossRef]

58. Salem, O.; Liu, Y.; Mehaoua, A.; Boutaba, R. Online anomaly detection in wireless body area networks for reliable healthcare monitoring. IEEE J. Biomed. Health 2014, 18, 1541-1551. [CrossRef] [PubMed]

59. Peng, Y.; Wang, X.; Guo, L.; Wang, Y.; Deng, Q. An efficient network coding-based fault-tolerant mechanism in WBAN for smart healthcare monitoring systems. Appl. Sci. 2017, 7, 817. [CrossRef]

60. Huang, H.; Gong, T.; Ye, N.; Wang, R.; Dou, Y. Private and secured medical data transmission and analysis for wireless sensing healthcare system. IEEE Trans. Ind. Inf. 2017, 13, 1227-1237. [CrossRef]

61. Usman, M.; Asghar, M.R.; Ansari, I.S.; Qaraqe, M. Security in wireless body area networks: From in-body to off-body communications. IEEE Access 2018, 6, 58064-58074. [CrossRef]

62. Angelidis, P. Monitoring quality-of-life parameters in wearable environments. Invent 2018, 3, 16. [CrossRef]

63. European Commission. https:/ / ec.europa.eu/commission/index_en (accessed on 7 August 2019).

64. European Commission. Smart Cyber-Physical Systems. Available online: https://ec.europa.eu/programmes / horizon2020/en/h2020-section/smart-cyber-physical-systems (accessed on 7 August 2019).

(C) 2019 by the authors. Licensee MDPI, Basel, Switzerland. This article is an open access article distributed under the terms and conditions of the Creative Commons Attribution (CC BY) license (http:/ / creativecommons.org/licenses/by/4.0/). 